\title{
Non-Concentric Multi-Compartment Fibers Fabricated Using a Modified Nozzle in Single-step Electrospinning
}

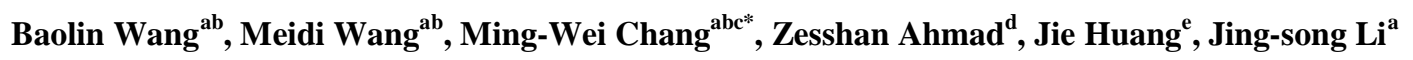

${ }^{\mathrm{a}}$ College of Biomedical Engineering \& Instrument Science, Zhejiang University, Hangzhou, 310027, China.

${ }^{\mathrm{b}}$ Zhejiang Provincial Key Laboratory of Cardio-Cerebral Vascular Detection Technology and Medicinal Effectiveness Appraisal, 310027, China.

${ }^{\mathrm{c}}$ Key Laboratory for Biomedical Engineering of Education Ministry of China, Hangzhou, 310027, China.

${ }^{\mathrm{d}}$ Leicester School of Pharmacy, De Montfort, University, The Gateway, Leicester, LE19BH, UK.

${ }^{\mathrm{e}}$ Department of Mechanical Engineering, University College London, London WC1E7JE, UK.

${ }^{*}$ Correspondence to: Ming-Wei Chang, Ph.D., Assoc. Professor,

$\underline{\text { Tel:+86(0)571-87951517,Email:mwchang@zju.edu.cn }}$

\begin{abstract}
Multi-compartment fibers with well-defined regions and functional media hosting potential provide coencapsulation opportunities for a variety of bio-molecules, drugs and even nutrients. In this research, fibers possessing multiple compartments were prepared using the single-step electrospinning method and a modified nonconcentric multi-needle. Polyvinyl pyrrolidone (PVP) was used as the fiber shell material, while ketoconazole (KCZ, model drug) and Sudan Red (model probe) were encapsulated as two separate segments running along the fiber length. Multi-compartment fiber morphology and structure were examined using optical and electron microscopy. The effect of flow rate on fiber morphology was also investigated and the release of encapsulated $\mathrm{KCZ}$ and Sudan Red was examined using UV spectroscopy. The results present an efficient and promising method to engineer multicompartment fibers in a single step for several biomedical applications in lieu.
\end{abstract}

Keywords: polymers; non-concentric; co-encapsulation; multi-compartment; fibre technology.

\section{Introduction}

Ongoing developments in advanced drug delivery systems have been the cornerstone of several pharmaceutical and bio-engineering remits for the last three decades and have focused on a host of novel engineering methods for polymeric excipient materials. Key properties of polymeric structures such as porosity, high surface-to-volume ratio, flexibility on several scales, morphology and structure size tailoring are a few drivers for their emergence as suitable 
drug encapsulating forms [1]. Although several methods (e.g. bubble electrospinning, bubbfil spinning and centrifugal electrospinning) [2-5] exist to engineer continuous or medicated (bio-active) polymeric fiber systems on a larger scale; the electrospinning (ES) method is probably the most facile and is operational at ambient conditions making it ideal for sensitive biomolecules or other biologics (e.g.cells and proteins)[6]. Furthermore, such single matrix-based drug delivery systems offer controlled and site-specific delivery of molecules of interest [7]. Several other challenges, currently being investigated using well established dosage forms, such as multiple drug loading and compartmentalization need to be explored further for these emerging fiber systems.

Complex fibers exhibiting multiple-compartment, therefore, provide a real opportunity to incorporate several actives and desired properties into one polymeric drug dosage system [8]. This is also increasingly important since the use of two or more drugs (e.g. targeting both symptom and underlying cause) is becoming more common and is also a major feature of polypharmacy [9]. To this end, several multi-compartment fibers have been explored on the reduced drug toxicity and improved biocompatibility via controlled drug release [10]. However, the coencapsulation of two drugs, or two model materials, into a fiber core which run throughout the fiber length in adjacent non-concentric fashion is rare and needs to be explored.

In this study a modified ES nozzle head was developed to enable multi-compartment fiber production in a single polymer engineering step via the ES technique. PVP is a synthetic, biocompatible and non-toxic polymer which has been widely applied as a pharmaceutical excipient [11]. KCZ is an active drug agent and has been utilized to treat superficial and internal fungal indications [12]. Multi-compartment fibers were prepared using PVP as the shell matrix material, with $\mathrm{KCZ}$ and Sudan Red formulations as two separate inner compartmentalized thread-like chambers. The effect of process parameters on fiber morphology and stability were explored. Drug and model probe release profiles were assessed.

\section{Material and methods}

\subsection{Solution preparation}

Polyvinyl pyrrolidone (PVP, $\mathrm{M}_{\mathrm{w}}=1.3 \times 10^{6} \mathrm{~g} / \mathrm{mol}$ ), Sudan Red and Tween 80 were purchased from Sigma-Aldrich, USA. Ketoconazole (KCZ, 99.7\%) was provided by Zhongtian Instrument, China. Phosphate buffer saline (PBS, pH7.4) and ethanol were supplied by Sinopharm Chemical, China. Dimethyl-silicone oil (viscosity 100 mPaS) was purchased from Aladdin chemistry, China. Deionized water was produced with a Milipore Milli-Q ultra-pure purifier (USA). PVP solutions were prepared by dissolving known quantities of PVP in ethanol through magnetic 
stirring(VELP-ARE magnetic stirrer, Italy) for $2 \mathrm{hrs}$ to form a concentration of $20 \% \mathrm{w} / \mathrm{v}$. Sudan Red and KCZ was dissolved in silicone oil separately at a concentration of $0.5 \% \mathrm{w} / \mathrm{v}$.

\subsection{Electrospinning process}

Multi-compartment fibers were generated using a dual-core shell ES set-up shown in Fig.1a. The set-up comprised a high voltage power supply (Glassman Inc, USA), three syringe pumps (KD Scientific KDS100, USA). Two nonconcentric metallic capillaries were located inside an outer enveloping metal needle to assemble a modified nozzle head. Silicone oil formulations of KCZ and Sudan Red were perfused into the two inner capillaries separately. PVP solution was infused into the outer needle. The electrically conductive modified nozzle was connected to the high power voltage supply.

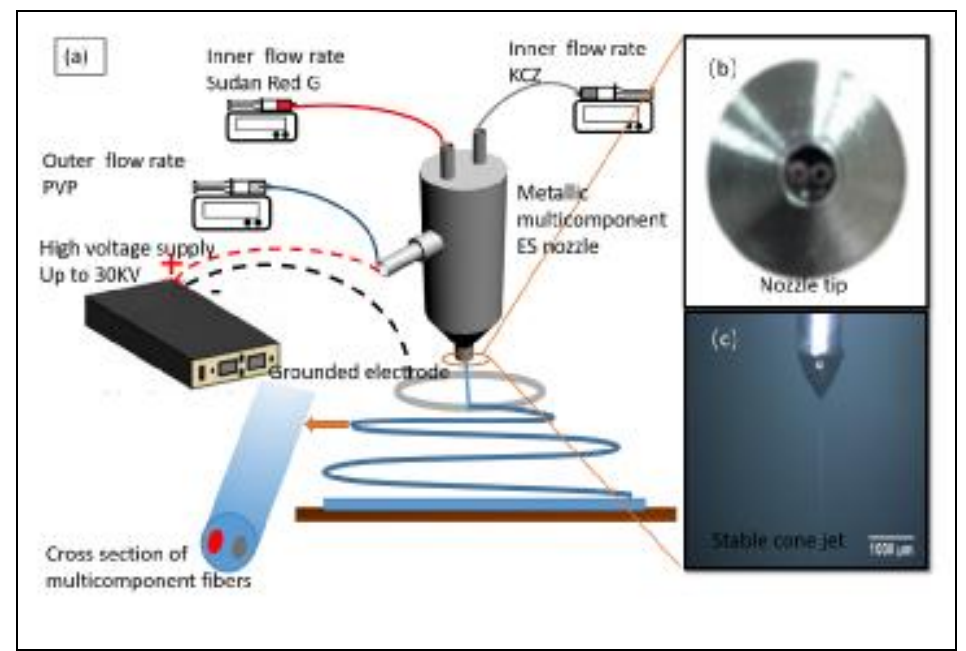

Figure 1.(a) A schematic diagram of the electrospinning set-up.(b)The modified nozzle head.(c)A digital image of a stable cone-jet captured during the electrospinning process.

\subsection{Characterization}

Optical (Pheonix-BMC503, China) and scanning electron microscopy (SEM, FEI Quanta650, Netherland) were used to study the morphology of multi-compartment fibers. The micrographs were subsequently analyzed using ImageJ software (National Institute of Health, USA) and fiber diameters were quantified using statistical distribution that involved a random sample of 50 fibers for each experimental condition. Error bars were plotted to represent the mean +/- standard deviation. Fourier transform infrared spectroscopy (FTIR, IR Affinity-1, Shimadzu, Japan) was utilized to determine chemical compositions, presence and stability of multi-compartment fibers at a resolution of $4 \mathrm{~cm}^{-1}\left(4700-400 \mathrm{~cm}^{-1}\right)$ using 20 scans. The hydrophobicity of multi-compartment fibers was assessed using an optical 
contact angle \& interface tension meter (SL2000KB, Kino Industry CO., USA). All data was exported for analysis and graphs were plotted using Origin software (OriginLab, USA).

\subsection{In-vitro drug release}

In-vitro release of model compounds (drug and probe) from multi-compartment fibers was measured using an UV spectrometer (UV-2600 spectrophotometer, Shimadzu, Japan) to determine the release profile of two different materials $[12,13]$. The release profiles for actives were measured using $100 \mathrm{ml}$ release medium with $30 \mathrm{mg}$ of each fiber sample type. The concentration of KCZ and Sudan Red in the supernatant was determined using UV absorption at a wavelength of 208 and 503nm respectively. Sample assessment was performed in triplicate.

\section{Results and discussion}

To enable continuous fabrication of fiber with well-defined multi-chambers, a modified ES system nozzle was used (Fig.1b). The critical voltage applied to an electrically conductive flowing medium to enable Taylor cone formation and a straight thinning jet $\left(\mathrm{V}_{\mathrm{c}}\right)$ is closely related to the sheath diameter of the loading nozzle comprising two nonconcentric metallic capillaries [14]. When the applied voltage reached $18 \mathrm{kV}$, the cone-jet was observed using a high-speed camera (Fig.1c). Most research utilizing ES systems focus on single matrix type materials with one inflowing medium [12]. In this study, several inflowing media were utilized and were also in non-concentric mode. The inner (non-centric needles hosting active and probe) flow rate limit was determined at $0.3 \mathrm{ml} / \mathrm{h}$. An increase from this value leads to droplet formation arising from inner fluid-jet break up due to insufficient PVP encapsulating capability. The lower limit was determined to be $0.18 \mathrm{ml} / \mathrm{h}$, and flow rates utilized below this value yielded non-continuous droplets embedded within the PVP fiber (Fig.2a). Once optimized (18.5kV, PVP: $6.5 \mathrm{ml} / \mathrm{h}$, both drug/probe:0.3ml/h), multiple-compartment fibers with two separate distinctive embedded threads spanning the entire fiber were observed (Fig.2b). Fig.2c shows a SEM of a uniform multi-compartment fiber with smooth surface topographies. 


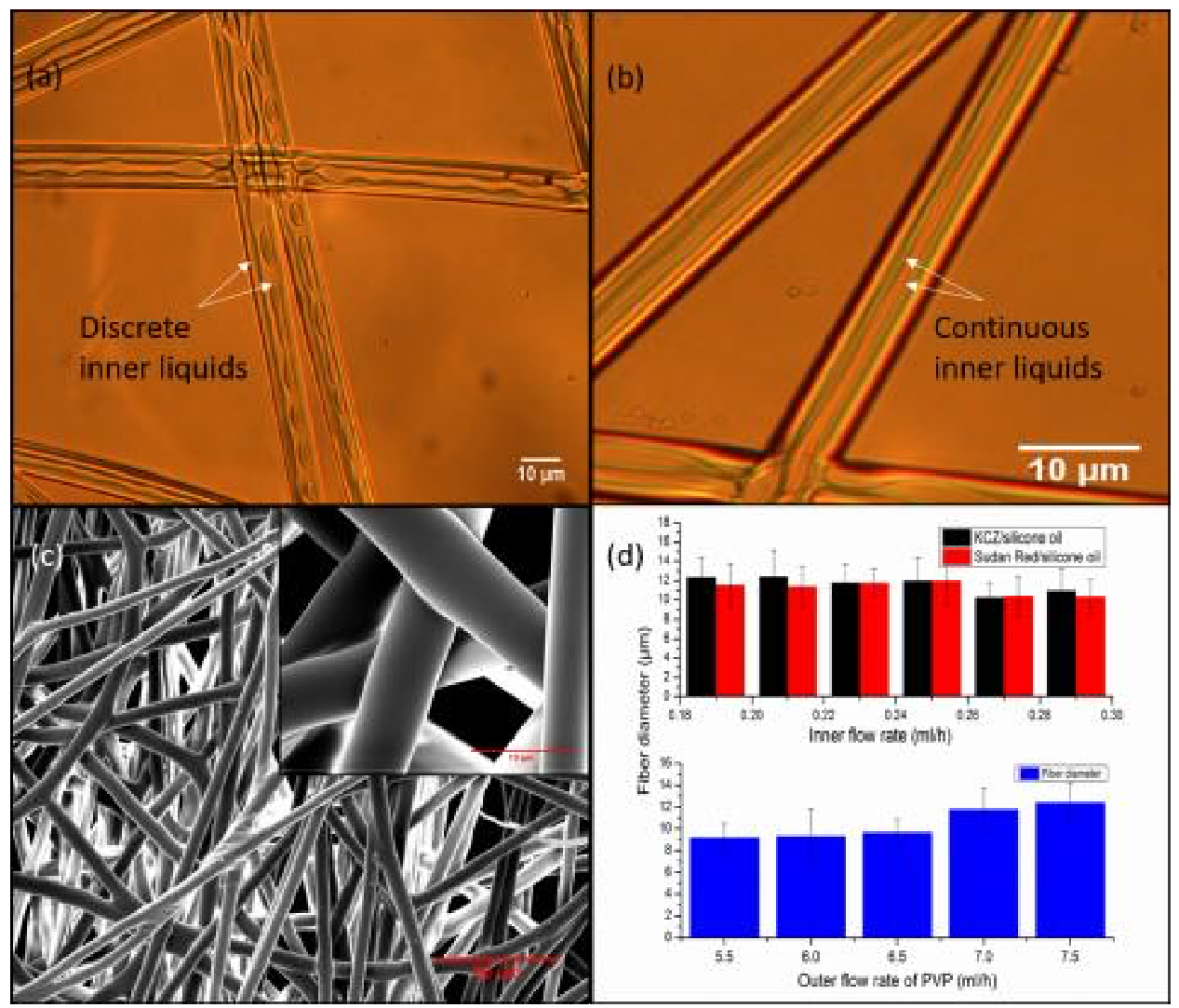

Figure 2.(a)An OM of a multi-compartment fiber showing discrete silicone oil distributions.(b)An OM of a multicompartment fiber exhibiting continuous oil encapsulation streams .(c)SEM of multi-compartment fibers.(d)Mean fiber diameter distribution in relation to inner and outer flow rates of selected media.

Process parameter exploration is a crucial aspect of ES and related technologies. In this study, selected inner media flow rates were varied between 0.19 to $0.29 \mathrm{ml} / \mathrm{h}$, while the applied voltage was fixed at $18 \mathrm{KV}$ and the outer flow rate was also constant $(5.5 \mathrm{ml} / \mathrm{h})$. The impact of inner medium flow rate (between optimized flows of 0.18 and $0.3 \mathrm{ml} / \mathrm{h}$ ) had little impact (Fig.2d). In contrast, when the outer medium flow rate is increased from 5.5 to $7.5 \mathrm{ml} / \mathrm{h}$, there is a clear difference in size (from 9 to $12 \mu \mathrm{m}$ ). This is due to the ratio of inflows (inner:outer).

The FTIR spectrum corresponding to pure PVP shows an absorbance at 1292 and $1018 \mathrm{~cm}^{-1}$, which is assigned to C$\mathrm{N}$ stretching (Fig.3a)[11]. The spectrum for silicone oil displays absorptions in the regions: 1260 and $1080 \mathrm{~cm}^{-1}$ due to $\mathrm{Si}-\mathrm{C}$ and $\mathrm{Si}-\mathrm{O}$ bands [12]. The characteristic absorption peaks of $\mathrm{KCZ}$ are 1512 and $1244 \mathrm{~cm}^{-1}$ [12].Similar bands are observed in the spectrum for multi-compartment fibers, suggesting the encapsulation of both KCZ and Sudan Red within the PVP matrix with no change to the chemical compositions. 
The contact angle of pure PVP membranes and multi-compartment fiber membranes were assessed and compared to investigate differences in hydrophilicity. Pure PVP fibrous membranes (Fig.3b) and multi-compartment fiber mats (Fig.3c) appear hydrophilic (water contact angles for neat PVP membranes and multi-compartment fibers were $79.1 \pm 0.2$ and $79.1 \pm 0.1^{\circ}$, respectively) and are in accordance with characteristic values of PVP samples reported previously [15]. The identical water contact angle values support perfect encapsulation of KCZ and Sudan Red as compartmentalized entities.

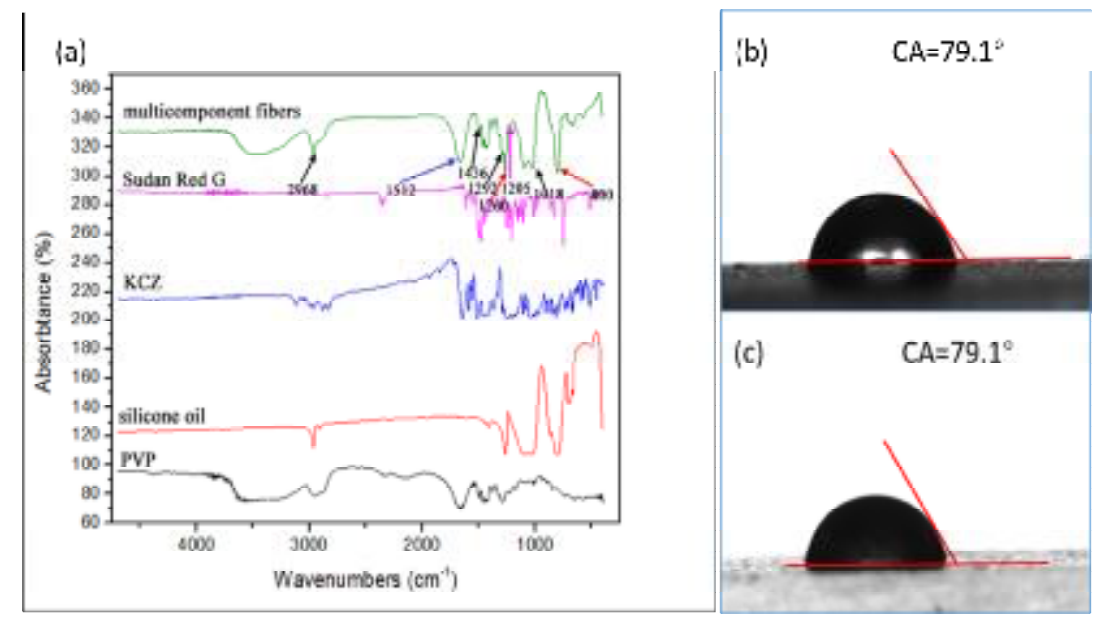

Figure 3. (a)FTIR spectra of pure materials and multi compartment fibers. Contact angles of water on (b) pure PVP membrane and (c) multi- compartment fibers.

Fig.4 shows release profiles of Sudan Red and KCZ (according to a previous method) [13]. Sudan Red and KCZ differ in their chemical composition and molecular weight, although both materials are sheathed simultaneously during ES. However, $\mathrm{KCZ}$ displays an initial burst release from the PVP matrix. In the first 5 minutes, more than $\sim 50 \pm 1.5 \%$ of encapsulated $\mathrm{KCZ}$ is released, followed by more sustained and slow, reaching $\sim 97 \pm 0.2 \%$ of $\mathrm{KCZ}$ at the 100 minutes time interval. The release behavior of Sudan Red is slightly more sustained in the first 5 minutes $(\sim 28 \pm 0.3 \%)$ and $\sim 80 \pm 0.8 \%$ is released at the 15 minutes interval. In general, the rapid release behavior of both compounds is attributed to the hydrophilic nature of PVP, which dissolves favorably in the dissolution medium (PBS). The burst release behavior is beneficial, as multi-compartment fibers have the potential to be utilized as wound dressing materials where drug needs to be released rapidly at the intended site [16]. 


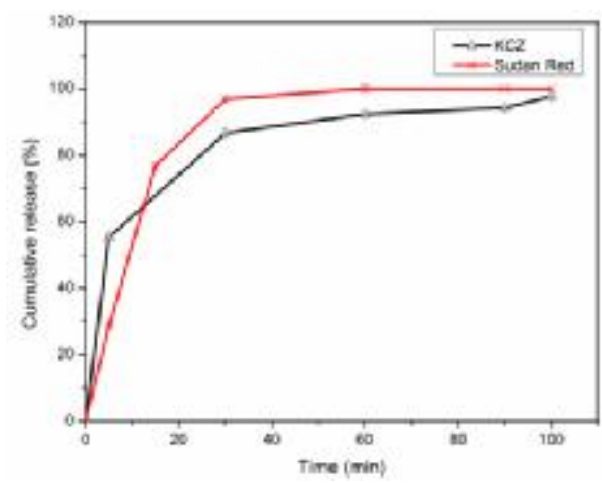

Fig. 4. Release profiles of $\mathrm{KCZ}$ and Sudan Red.

\section{Conclusions}

Multi-compartment fibers with well-defined inner compartments were successfully prepared using a modified nozzle head and ES technique. The co-encapsulation of two compounds (in non-concentric) was generated. The effect of inner and outer media flow rate on the mean fiber diameter indicates the outer medium drives fiber morphology due to the increased infusion rate. Multi-compartment fibers appear hydrophilic and exhibit near identical contact angles to that of pure PVP fibrous membranes. Release of KCZ and Sudan Red suggests the potential of co-encapsulating a variety of agents into a single fiber matrix with broader implications in drug delivery and biomedical research fields.

\section{Acknowledgements}

This work was financially supported by the Key Technologies R\&D Program of Zhejiang Province(2015C02035) and the Fundamental Research Funds for the Central Universities.

\section{References}

[1] Xu J, Jiao Y, Shao X, Zhou C. Controlled dual release of hydrophobic and hydrophilic drugs from electrospun poly( I -lactic acid) fiber mats loaded with chitosan microspheres. M ater Lett. 2011;65:2800-3.

[2] Yang R, He J, Xu L, Yu J. Bubble-electrospinning for fabricating nanofibers. Polymer. 2009;50:5846-50.

[3] Liu P, Cai JJ, Zhao L, He JH. Micro-nanofibers with hierarchical structure by bubbfil-spinning. Therm Sci. $2015 ; 19: 1455-6$.

[4] Wang L, Chang MW, Ahmad Z, Zheng H, Li JS. M ass and controlled fabrication of aligned PVP fibers for matrix type antibiotic drug delivery systems. Chem Eng J. 2016;307:661-9.

[5] Zhang C, Gao C, Chang M W, Ahmad Z, Li JS. Continuous micron-scaled rope engineering using a rotating multinozzle electrospinning emitter. Appl Phys Lett. 2016;109:173105-18897. 
[6] Toniatto TV, Rodrigues BVM, Marsi TCO, Ricci R, Marciano FR, Webster TJ, et al. Nanostructured poly (lactide acid) electrospun fiber with high loadings of TiO 2 nanoparticles: Insights into bactericidal activity and cell viability. Mat Sci Eng C-Mater. 2016;71:381-5.

[7] Yao Z-C, Gao Y, Chang M-W, Ahmad Z, Li J-S. Regulating poly-caprolactone fiber characteristics in-situ during one-step coaxial electrospinning via enveloping liquids. M aterials Letters. 2016;183:202-6.

[8] Natu MV, de Sousa HC, Gil MH. Effects of drug solubility, state and loading on controlled release in bicomponent electrospun fibers. Int J Pharm. 2010;397:50-8.

[9] Yu DG, Gao LD, White K, Branford-White C, Lu WY, Zhu LM. Multicomponent amorphous nanofibers electrospun from hot aqueous solutions of a poorly soluble drug. Pharm Res. 2010;27:2466-77.

[10] Hong KH, Park JL, Sul IH, Youk JH, Kang TJ. Preparation of antimicrobial poly(vinyl alcohol) nanofibers containing silver nanoparticles. J Polym Sci, Part B: Polym Phys. 2006;44:2468-74.

[11] Wang JC, Chang MW, Ahmad Z, Li JS. Fabrication of patterned polymer-antibiotic composite fibers via electrohydrodynamic (EHD) printing. J Drug Deliv Sci Tec. 2016;35:114-23.

[12] Wang BL, Zheng HX, Chang MW, Ahmad Z, Li JS. Hollow polycaprolactone composite fibers for controlled magnetic responsive antifungal drug release. Colloids Surf, B. 2016;145:757-67.

[13] Yuan G, Ding Z, Chang M W, Ahmad Z, Li JS. Optimising the shell thickness-to-radius ratio for the fabrication of oil-encapsulated polymeric microspheres. Chem EngJ. 2016;284:963-71.

[14] Yu DG, Yu JH, Chen L, Williams GR, Wang X. Modified coaxial electrospinning for the preparation of highquality ketoprofen-loaded cellulose acetate nanofibers. Carbohydr Polym. 2012;90:1016-23.

[15] Dahlberg C, Millqvist-Fureby A, Schuleit M, Furo I. Polymer-drug interactions and wetting of solid dispersions. Eur J Pharm Sci. 2010;39:125-33.

[16] Carson D, Jiang Y, Woodrow KA. Tunable Release of Multiclass Anti-HIV Drugs that are Water-Soluble and Loaded at High Drug Content in Polyester Blended Electrospun Fibers. Pharm Res. 2015;33:125-36. 